\title{
Tratamiento de la estenosis valvular aórtica severa degenerativa: Actualidad y futuro. Perspectiva del cirujano cardíaco
}

\author{
Severe aortic stenosis treatment in the elderly: present and future. \\ The cardiac surgeon perspective
}

\author{
Daniel Vásquez Echeverri, Francisco Estévez-Cid, José J. Cuenca Castillo \\ Servicio de Cirugía Cardíaca. Complejo Hospitalario Universitario A Coruña. SERGAS. A Coruña
}

\section{Introducción}

La estenosis valvular aórtica (EA) es la valvulopatía degenerativa más prevalente en el mundo occidental, con una incidencia de $12,4 \%$ en pacientes mayores de 75 años ${ }^{1}$ El principal mecanismo de estenosis consiste en la calcificación y fusión de los velos y el anillo aórtico debido a un proceso degenerativo. Entre los factores de riesgo que se asocian a la EA se encuentran la edad, sexo masculino, hipercolesterolemia, insuficiencia renal, hipertensión arterial, diabetes y tabaco entre otros². También se asocia frecuentemente a válvulas aorticas bicúspides, que se encuentran hasta en el $60 \%$ de los pacientes con EA menores de 70 años ${ }^{3,4}$.

Según algunas series, la mortalidad de esta patología una vez existen síntomas se eleva hasta el $50 \%$ al año, y del $80 \%$ a los 2 años, por lo que es importante el diagnóstico precoz e implica un seguimiento estricto de estos pacientes ${ }^{5,6}$. Estudios realizados en subpoblaciones mayores de 80 años con EA severa sintomática han demostrado que la sustitución valvular reduce la mortalidad al año y a los 5 años a $13 \%$ y $32 \%$ respectivamente ${ }^{7}$. Sin embargo solo entre el $50 \%$ y el $70 \%$ de los pacientes se llegan a operar, debido a que una parte importante de esta población es de edad avanzada y tiene múltiples comorbilidades, que hacen que se desestimen para cirugía convencional por su elevado riesgo quirúrgico $0^{8-10}$.

El diagnóstico de la EA es sencillo con una adecuada anamnesis y exploración física sistmática. No hay que olvidar la triada sintomática clásica de EA: angina, disnea y síncope. Se debe, por tanto, auscultar a todo paciente que presente cualquiera de estos síntomas en busca de soplos y realizar un ecocardiograma para confirmar el diagnóstico y valorar su severidad (Tabla1). Un electrocardiograma con signos de hipertrofia ventricular izquierda nos puede hacer sospechar de esta patología en pacientes asintomáticos.

\section{Indicación quirúrgica}

Las guías actuales sobre el diagnóstico y tratamiento de valvulopatías en adultos de la Sociedad Europea de Cardiología ESC (2012) y las guías de la American Heart Association/ American Collegue of Cardiology AHA/ACC (2014), coinciden en la indicación quirúrgica de la EA severa sintomática como recomendación I con nivel de evidencia $A^{11,12}$. También está indicada la cirugía en pacientes asíntomaticos con EA
Tabla 1. Clasificación de la estenosis aórtica (modificado de Nishimura RA et al12).

\begin{tabular}{|c|c|c|}
\hline Estadio & Descripción & Definición \\
\hline A & En riesgo & $\begin{array}{l}\text { Esclerosis valvular } 0 \text { válvula aórtica } \\
\text { bicúspide; } V \max <2 \mathrm{~m} / \mathrm{seg}\end{array}$ \\
\hline B & Progresiva & $\begin{array}{l}\text { Calcificación valvular ligera a moderada, } 0 \\
\text { cambios reumáticos con disminución de } \\
\text { movilidad de velos; } \\
\text { Vmax 2-3,9m/seg } \\
\text { Gradiente medio } 20-39 \mathrm{mmHg}\end{array}$ \\
\hline C1 & $\begin{array}{l}\text { Estenosis aórtica } \\
\text { severa asintomática } \\
\text { con FEVI } \\
\text { conservada. }\end{array}$ & $\begin{array}{l}\text { Calcificación valvular severa o cambios } \\
\text { reumáticos con movilidad de velos } \\
\text { reducida; } \\
\text { Vmax }>4 \mathrm{~m} / \mathrm{seg} \\
\text { Gradiente medio }>40 \mathrm{mmHg} \\
\mathrm{FEVI}>50 \%\end{array}$ \\
\hline $\mathrm{C} 2$ & $\begin{array}{l}\text { Estenosis aórtica } \\
\text { severa asintomática } \\
\text { con FEVI disminuida }\end{array}$ & $\begin{array}{l}\text { Calcificación valvular severa o cambios } \\
\text { reumáticos con movilidad de velos } \\
\text { reducida; } \\
\text { Vmax }>4 \mathrm{~m} / \text { seg; } \\
\text { Gradiente medio > 40mmHg; } \\
\mathrm{FEVl}<50 \%\end{array}$ \\
\hline D1 & $\begin{array}{l}\text { Estenosis aórtica } \\
\text { severa sintomática } \\
\text { con gradientes } \\
\text { elevados }\end{array}$ & $\begin{array}{l}\text { Calcificación valvular severa o cambios } \\
\text { reumáticos con movilidad de velos } \\
\text { reducida; } \\
\text { Vmax }>4 \mathrm{~m} / \mathrm{seg} \text {; } \\
\text { Gradiente medio }>40 \mathrm{mmHg}\end{array}$ \\
\hline D2 & $\begin{array}{l}\text { Estenosis aórtica } \\
\text { severa sintomática } \\
\text { con bajo gradiente y } \\
\text { FEVI disminuida }\end{array}$ & $\begin{array}{l}\text { Calcificación valvular severa o cambios } \\
\text { reumáticos con movilidad de velos } \\
\text { reducida; } \\
\text { Basal: área valvular aórtica }<1 \mathrm{~cm} 2 \text {, Vmax } \\
<4 \mathrm{~m} / \mathrm{seg} \text {, FEVI }<50 \% \text {; } \\
\text { Post stress test con dobutamina: } \\
\text { área valvular aórtica }<1 \mathrm{~cm} 2 \text {, Vmax }>4 \mathrm{~m} / \\
\text { seg }\end{array}$ \\
\hline D3 & $\begin{array}{l}\text { Estenosis aórtica } \\
\text { severa sintomática } \\
\text { con bajo gradiente, } \\
\text { bajo flujo, y FEVI } \\
\text { conservada. }\end{array}$ & $\begin{array}{l}\text { Calcificación valvular severa o cambios } \\
\text { reumáticos con movilidad de velos } \\
\text { reducida; } \\
\text { área valvular aórtica }<1 \mathrm{~cm} 2 \text {, Vmax }<4 \mathrm{~m} / \\
\text { seg, FEVI }>50 \% \text {, área valvular aórtica } \\
\text { indexada }<0.6 \mathrm{~cm} 2 / \mathrm{m} 2 \text {, volumen latido } \\
\text { indexado }<35 \mathrm{ml} / \mathrm{m} 2 \text {. }\end{array}$ \\
\hline
\end{tabular}

Vmax: Velocidad máxima; FEVI: Fracción de eyección de ventrículo izquierdo. 
severa si tienen disminución de la función venticular izquierda (FEVI $<50 \%)$, o van a ser intervenidos por otra indicación, como la revascularización quirúrgica (Recomendación IA). La sustitución valvular aórtica convencional (SVA) en pacientes con EA moderada, sólo está indicada en pacientes sintomáticos con disfunción ventricular izquierda ( $\mathrm{FEVI}<50 \%) 0$ en el caso de que, por datos hemodinámicos y clínicos, se demuestre que la estenosis valvular sea la causa más probable de los síntomas. No está indicada la cirugía en pacientes con EA ligera o moderada asintomáticos.

En los pacientes con indicación de SVA, el siguiente paso consiste en valorar si se beneficiarán de una intervención quirúrgica, es decir, si al corregir la valvulopatía, el paciente va a mejorar su clase funcional y su calidad de vida. Si la respuesta es afirmativa, se debe evaluar a continuación el riesgo quirúrgico del paciente. Para ello, las dos herramientas ampliamente aceptadas para valorar de forma objetiva el riesgo de mortalidad quirúrgica son la escala de riesgo de la Sociedad de Cirujanos Torácicos (STS-PROM) y el EUROSCORE logístico. Estas escalas tienen en cuenta múltiples variables como son la edad, género, tipo de cirugía y comorbilidades asociadas, entre otras; y nos ayuda a estimar el riesgo quirúrgico como alto (STS score > 10\%; EUROSCORE logístico > 20\%), intermedio (STS score 4-8\%; EUROSCORE 10-20\%) o bajo. Se considera que la cirugía está contraindicada cuando la mortalidad o morbilidad irreversible es mayor al $50 \%$. Hay que tener presente que estas escalas no han sido diseñadas específicamente para subpoblaciones de alto riesgo con EA y no deben ser la única herramienta para definir el riesgo quirúrgico de un paciente. Se ha demostrado que el EUSCORE logístico, y en menor medida el STS, suelen sobreestimar la mortalidad en pacientes con múltiples comorbilidades, por lo que sólo deben de ser usadas como una referencia (ambas escalas están disponibles en la web en: riskcalc.sts.org y www.euroscore.org) ${ }^{13,14}$. La mortalidad global de la SVA convencional para EAo aislada reportada por la Sociedad Española de Cirugia Torácica y Cardiovascular durante el año 2012, incluyendo condiciones de emergencia por inestabilidad hemodinámica e infección, fué de $4,1 \%$ (frente al $8,1 \%$ de la esperada por la escala Euroscore $^{15}$. En poblaciones seleccionadas de menor edad y mayor expectativa de vida, se han reportado en los registros una tasas de mortalidad y complicaciones mayores por debajo del $2 \%{ }^{16}$

Por otra parte, hay que tener presente que existen ciertas comorbilidades que pueden suponer una contraindicación, al menos relativa, para la cirugía cardiaca convencional, como por ejemplo: pacientes con cirugía cardiaca previa e injertos coronarios permeables, pacientes con EPOC muy severo, pacientes obesos con IMC $>40$, pacientes que han recibido radioterapia en el tórax, pacientes con aorta severamente calcificada ("en porcelana"), en los que al estar muy calcificada la aorta no permite clamparla, y pacientes con criterios de fragilidad. La fragilidad se define como un síndrome de disminución de la reserva fisiológica y resistencia al stress, que conlleva una mayor vulnerabilidad y aparición de efectos adversos en diferentes órganos y sistemas ${ }^{17,18}$. Se han propuesto varias escalas para medir la fragilidad de los pacientes ${ }^{18}$. Algunas de ellas tienen en cuenta la distancia que puede recorrer un paciente en 6 minutos, si han perdido peso de forma involuntaria, la fuerza prensil de las manos, niveles séricos de albúmina y las actividades de la vida diaria que son capaces de realizar sin ayuda, entre otras. Sin embargo, en la actualidad la graduación de la fragilidad es un tema controvertido y no existe aún un claro consenso ampliamente aceptado sobre el mismo. En cualquier caso, lo cierto es que existen múltiples factores tanto técnicos como clínicos, que no ponderan claramente las escalas objetivas de riesgo quirúrgico ${ }^{18}$. Por tanto, todos los grupos y guías de actuación coinciden en apuntar la importancia de que pacientes con alto o muy alto riesgo quirúrgico sean comentados en una sesión clínica compuesta por el llamado "HEART TEAM"4,17. La fragilidad siempre debe ser valorada por este equipo HEART TEAM, y se decidirá de forma consensuada cuando un paciente es muy frágil por lo que se le indicará una TAVI, o que es demasiado frágil y se contraindicará la cirugía ${ }^{17,19}$.

EI HEART TEAM debe estar compuesto al menos por un cirujano cardiaco, un cardiólogo clínico o el clínico responsable del paciente, un cardiólogo intervencionista, un especialista en imagen y un anestesista con experiencia en cirugía cardiaca. (Recomendación IC). En esta sesión clínica se discutirá la indicación quirúrgica de cada paciente, y se tendrán en cuenta factores de riesgo, clase funcional basal y esperada después de la cirugía, fragilidad, e incluso el soporte social de cada paciente. Se evaluarán las imágenes diagnósticas realizadas y se discutirán posibles complicaciones o dificultades técnicas esperadas en base a la experiencia de cada centro. Así se decidirá por consenso cual es la mejor estrategia terapéutica para cada paciente. Actualmente, además de la SVA, disponemos de alternativas menos invasivas como la SVA por miniesternotomia o el implante valvular aórtico transcatéter (TAVI). También existen nuevos tipos de válvulas como las "sutureless", que están diseñadas para anclarse en la raíz aórtica sin necesidad de suturas, por lo que acortan de forma significativa el tiempo de circulación extracorpórea, especialmente aptas para su implante por miniesternotomia.

\section{Sustitución valvular aórtica: Abordajes quirúrgicos y tipos de válvulas Esternotomía media y miniesternotomía}

Entendemos por cirugía de SVA convencional aquella en que se accede al mediastino mediante esternotomía media. Como ventaja ofrece una excelente exposición del corazón y los grandes vasos, lo que permite una cirugía cómoda puesto que permite un amplio exposición del campo quirúrgico. Es la técnica más difundida, con una curva de aprendizaje muy corta y reproducible. Una desventaja de este abordaje consiste en que, dado que se secciona por completo el es- 
ternón, genera una mayor inestabilidad esternal en el postoperatorio inmediato y mayor dolor, lo que puede retrasar la rehabilitación respiratoria y la estancia postoperatoria. Como alternativa menos invasiva a este abordaje existe la miniesternotomía superior en "J", en la que sólo se secciona parcialmente el esternón. De esta forma se consigue una óptima exposición de la aorta ascendente, y un adecuado campo quirúrgico para realizar la sustitución valvular con seguridad. Con esta técnica se permite lograr una mayor estabilidad esternal, y por lo tanto una mejor mecánica respiratoria y mayor confort en el postoperatorio inmediato, lo que repercute en un alta hospitalaria más precoz ${ }^{16,20-22}$. Está técnica es especialmente recomendable en pacientes mayores, en los que se ha asociado una menor necesidad de transfusión de hemoderivados, y con patología pulmonar, en los que la fisioterapia respiratoria tiene un papel determinante en la recuperación ${ }^{22}$. Con la miniesternotomía, además, se consigue un efecto cosmético favorable, ya que se emplean pequeñas incisiones en la piel de sólo 8 centímetros de longitud. En el Complejo Hospitalario Universitario A Coruña (CHUAC), desde el año 2011 empleamos este abordaje miniinvasivo en más de 120 pacientes con excelentes resultados funcionales y por qué no decirlo, cosméticos (Figura 1).

\section{Prótesis valvulares sin sutura 0 de rápida liberación}

Las denominada prótesis valvulares sin sutura 0 "sutureless" (Perceval, Sorin Group ${ }^{\circledR}$; Intuity Elite Valve, Edwards Lifesciences $^{\circledR}$; 3F Aortic Bioprosthesis, Medtronic ${ }^{\circledR}$ ), son un nuevo tipo de prótesis biológicas que se implantan en el anillo aórtico sin necesidad de puntos de sutura, de ahí su nombre (Figura 2). Éstas se encuentran montadas en un stent que se expande y fija en el anillo aórtico tras su decalcificación, mediante liberación controlada bajo visión directa. Una de las principales ventajas de estas prótesis es el impacto en el menor tiempo de implante, con lo que se acorta significativamente el tiempo de pinzamiento aórtico y circulación extracorpórea (disminuyendo la incidencia de sus efectos adversos); y favoreciendo las técnicas de implante miniinvasivas. Además, presentan un excelente comportamiento hemodinámico (gradientes valvulares muy bajos y tasas de fuga paravalvular por debajo del $2 \%)^{23,24}$. En el CHUAC, desde el año 2013 combinamos este tipo de
Figura 1. Herida quirúrgica al mes del recambio valvular aórtico mediante miniesternotomía superior (A) y esternotomía media convencional (B).

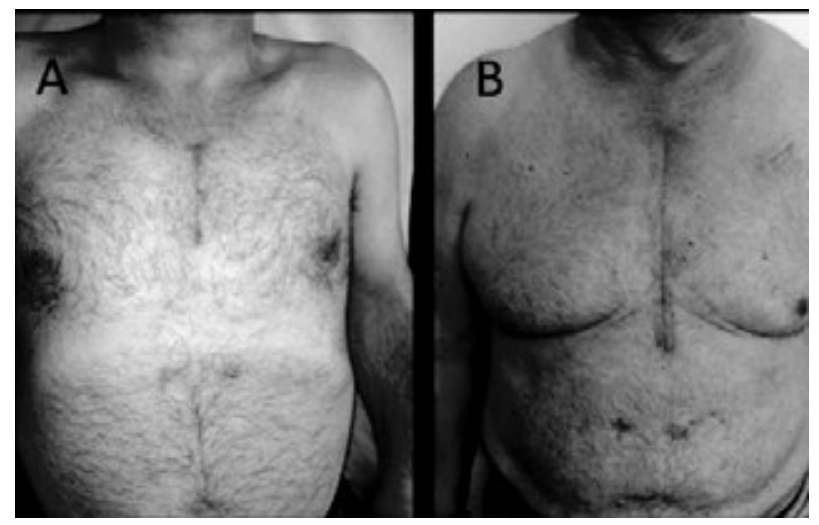

prótesis "sutureless" con un abordaje por miniesternotomía superior (estudio PERMS: PERceval MinieSternotomy), con lo que obtenemos un doble beneficio: reducción a la mitad del tiempo de circulación extracorpórea y un abordaje menos invasivo, que favorece una rápida recuperación postoperatoria. Se benefician de esta estrategia sobre todo pacientes mayores y con múltiples comorbilidades ${ }^{25-27}$.

\section{Prótesis valvulares aórticas: ¿biológicas o mecánicas?}

Está bien establecido en las guías clínicas la recomendación genérica de prótesis valvulares aórticas mecánicas en pacientes de hasta 60 años, así como el implante de prótesis biológicas en pacientes mayores de 70 años (Nivel de recomendación II-B) ${ }^{11}$. Sin embrago existen varias excepciones a la regla en este aspecto. Es importante tener en cuenta aspectos específicos de cada paciente como lo son la actividad física del paciente, su profesión, o el cumplimiento terapéutico esperado. Aún en pacientes jóvenes, si estos tienen un pobre soporte social, 0 una inadecuada adherencia al tratamiento, puede ser contraproducente implantar una válvula mecánica, que precisa de un control estricto del INR y una cierta disciplina. En el caso de pacientes con una vida activa 0 actividades de riesgo por su profesión, puede ser igualmente desaconsejable una prótesis que requiera de anticoagulación. Lo mismo ocurre en mujeres en edad fértil que desean tener hijos. Todas estas son variables a tener en

Figura 2. Bioprótesis valvulares aórticas sin sutura (sutureless) o de rápida liberación: Perceval, Sorin Group® (A); Intuity Elite Valve, Ed
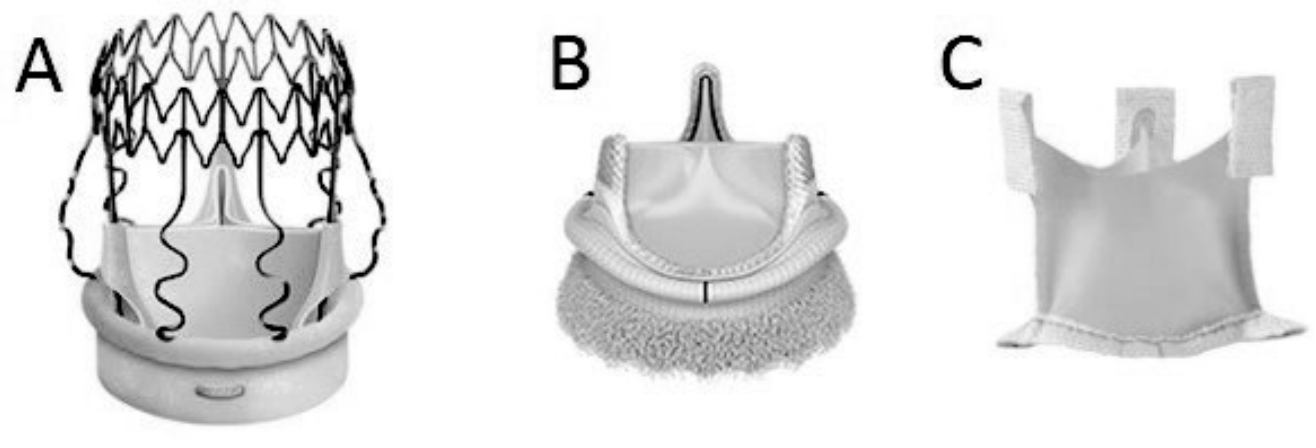
cuenta y que deben plantearse al paciente, quien ha de ser el que tome la decisión sobre el tipo de prótesis que desea. La durabilidad de las prótesis biológicas depende de muchos factores, de entre ellos quizás el más importante sea la edad del paciente al momento del implante. En este sentido, cuanto más edad tenga el paciente al momento del implante, mayor suele ser la durabilidad de las prótesis biológicas. En general, suele ser necesaria la reoperación entre los 7 y 12 años, dependiendo de la edad del paciente ${ }^{28}$.

\section{Implante valvular aórtico transcatéter - TAVI}

El implante valvular aortico transcatéter TAVR/TAVI, por sus siglas en inglés (Transcatheter Aortic Valve Replacement/Implantation), surge en la última década como una alternativa terapéutica para pacientes con EA severa sintomática y con muy alto riesgo quirúrgico que contraindica la intervención quirúrgica convencional.

Se trata de una técnica poco invasiva, en la que se introduce por vía transfemoral (a través de la arteria femoral común), 0 transapical (a través del ápex cardiaco, por minitoracotomía izquierda), una válvula aórtica biológica que se despliega, mediante control angiográfico y ecocardiográfico, por dentro de la válvula aórtica calcificada del paciente, resolviendo así las estenosis (Figura 3). Es por tanto un implante dentro de la válvula aórtica nativa del paciente, no una sustitución valvular. También es posible el implante por vía transaórtica (a través de la aorta ascendente), y por vía arterial axilar o subclavia, pero es un abordaje menos empleado. Este procedimiento se puede realizar en una sala de hemodinámica 0 idealmente en un quirófano de cirugía cardiaca bajo anestesia general, y no precisa el uso de circulación extracorpórea.

El primer implante de una válvula aortica transcateter se realizó el 16 de abril de 2002 (Cribier et al ${ }^{29}$ ) y desde 2007, las 2 válvulas aprobadas en Europa de mayor uso son la válvula Sapien (Edwards Sapien, Edwards Lifescience inc ${ }^{\circledR}$, California, Estados Unidos) y la CoreValve (Revalving System CoreValve ${ }^{\circledR}$, Paris, Francia; Medtronic, Minneapolis, EEUU) (Figura 4). La primera es una válvula de pericardio bovino con soporte de cromocobalto, que se ancla en el anillo aórtico y se expande mediante un un balón a presión con solución salina con un volumen calculado previamente según el diámetro de la válvula y el anillo aórtico de paciente. La segunda es una válvula autoexpandible de pericardio porcino con soporte de nitinol, que se ancla en el tracto de salida del ventrículo izquierdo. En los últimos años se han desarrollado nuevos modelos llamados "de segunda generación" como son la Sapien 3 (Edwards Lifescience inc. ${ }^{\circledR}$, California, EEUU), la Jena Valve (Jena Valve $^{\circledR}$, Munich, Alemania) la Symetis Accurate (Symetis SA ${ }^{\circledR}$, Ecublens, Suiza), y la Engager (Medtronic ${ }^{\circledR}$, Minnesota, EEUU) entre otras. Estos últimos modelos tienen en común un introductor más pequeño que facilita su implante por vía femoral, un sistema de recaptura, que permite un implante más preciso, y diferentes sistemas "anti- leaks" que han disminuido la incidencia de fugas periprotésicas. Se trata, por tanto, de un avance muy importante en los principales puntos débiles de este tipo de prótesis, sin embargo la mayoría se encuentran aún en fase de pruebas ${ }^{14}$.

El estudio PARTNER I (Placemenet of Aortic Transcatheter Valves), fue el primer estudio clínico aleatorizado sobre el implante de éstas válvulas, y se dividió en dos cohortes. La cohorte A aleatorizó pacientes operables, pero con muy alto riesgo quirúrgico, a TAVI o sustitución valvular aortica convencional, y no halló diferencias estadísticamente significativas a 1 año en relación a mortalidad, por lo que se consideró no inferior (24.2\% vs $26.8 \%$, respectivamente). La cohorte B aleatorizó pacientes inoperables a TAVI o tratamiento médico convencional y halló una reducción absoluta de la mortalidad a 1 año del $20 \%$ (30.7\% vs 50.7\%), por lo que se consideró superior al tratamiento médico ${ }^{30}$.

Actualmente este tipo de válvulas sólo están indicadas en pacientes con EA severa sintomática con un riesgo quirúrgico prohibitivo y una expectativa de vida mayor de 12 meses (Recomendacion IB), pacientes con EA severa sintomáticos y con un alto riesgo quirúrgico (recomendación IIB), o pacientes que el HEART TEAM considere que por su comorbilidades, aspectos técnicos específicos (aorta en porcelana, pacientes reoperados con injertos permeables...) o fragilidad, se beneficien de una TAVI.

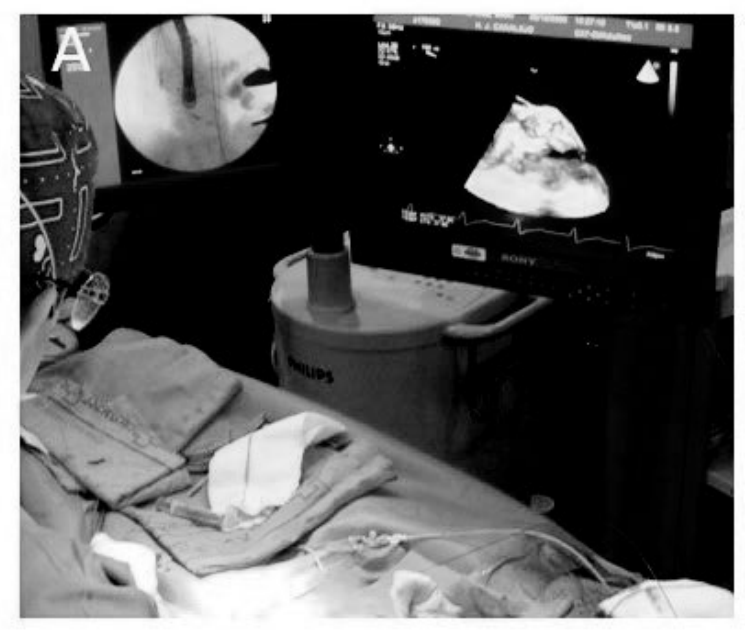

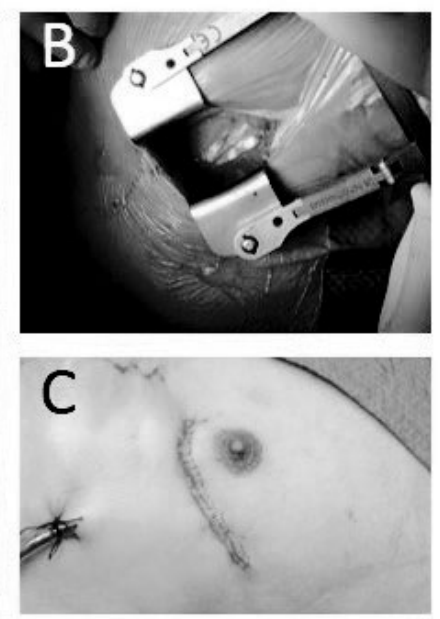

Figura 3. Implante de bioprótesis valvular áortica transcatéter vía transapical. La liberación protésica se realiza bajo control angiográfico y ecocardiografía-3D (A). El abordaje quirúrgico se realiza mediante minitoracotomía anterior izquierda accediendo al ápex cardíaco (B), precisándose incisiones cutáneas menores de $6 \mathrm{~cm}(\mathrm{C})$. 
Figura 4. Bioprótesis valvulares aórticas transcatéter: Edwards Sapien, (Edwards Lifescience $($ ) $(A)$ y CoreValve (Medtronic () ) (B).
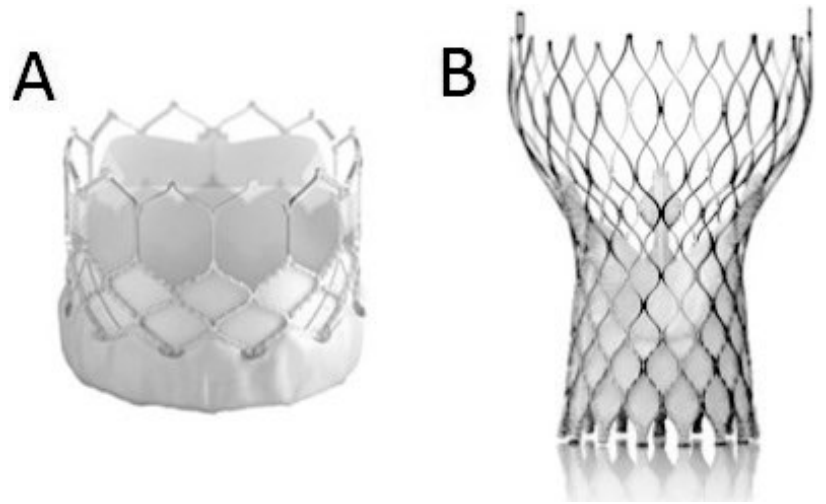

Están actualmente en curso dos estudios clínicos aleatorizados que incluyen pacientes con riesgo intermedio; el "Surgical Replacement and transcatheter Aortic Implantation -SURTAVI TRIAL" (Corevalve ${ }^{\circledR}$; Medtronic), y el "Placement of Aortic Transcatheter Valve (PARTNER II TRIAL, Sapien XT ${ }^{\circledR}$; Edwars). Sin duda, los resultados de estos estudios son muy esperados y es posible que en un futuro próximo amplíen las indicaciones de las TAVI.

La vía de abordaje, transfemoral, transapical o transaórtica dependerá de la anatomía del paciente, y de la experiencia de cada centro. Inicialmente sólo se disponía de introductores valvulares relativamente grandes (22Fr - 25Fr) por lo que si el paciente tenia enfermedad arterial periférica, 0 una anatomía poco favorable, dado por oclusión o tortuosidad el eje arterial ileofemoral, no era posible el abordaje transfemoral y se utilizaba la vía transapical. Esta estrategia, conocida como "transfemoral first", llevó a que los pacientes operados por vía transapical tuvieran todos un mayor riesgo quirúrgico, al tener más comorbilidades, por lo que tenían una mayor mortalidad. Sin embargo, con las válvulas transcateter de última generación, con un diámetro de 18Fr, se han igualado cada vez más ambos grupos. La decisión del abordaje depende ahora más de la experiencia del centro y de las demás comorbilidades del paciente. El centro que implante éste tipo de válvulas siempre deberá tener la disponibilidad de un servicio de cirugía cardiaca para tratar eventuales complicaciones.

Las principales complicaciones médico-quirúrgicas para ambos tipos de prótesis valvulares y abordajes son accidentes cerebrovasculares $(3.3 \%)$, fugas paravalvulares significativas (15-20\%), oclusión de arterias coronarias $(0.6 \%)$, bloqueo aurículo-ventricular que precise implante de marcapasos definitivo (6.5-28\%) y complicaciones vasculares tipo disección 0 trombosis arteriales $(18 \%)^{31-35}$. Sin duda, el avance tecnológico progresivo y la experiencia adquirida contribuirán a un marcado descenso en este tipo de complicaciones ${ }^{36}$.

\section{TAVIS en Europa y España}

Desde su comercialización en 2007 se han implantado más de 100.000 TAVIs en el mundo ${ }^{37}$. Actualmente es Alemania el país de Europa donde más se implantan este tipo de válvulas, siendo responsable del $45,9 \%$ de todos los implantes, seguida de Italia $(14,9 \%)$ y Francia $(12,9 \%)^{38}$. Desde 2008 se han implantado en el CHUAC más de 200 TAVIs (transapicales y transfemorales) con muy buenos resultados, similares a los reportados en otras series. La edad media de los pacientes operados en nuestro centro mediante TAVI transapical es de 81 años, con similar proporción entre hombres y mujeres (57\% y $43 \%$ respectivamente), y una media de estancia hospitalaria de 7,5 días.

\section{Coste efectividad y calidad de vida}

Debido al alto coste de este tipo de prótesis, que es seis veces superior al de una válvula aórtica convencional, existe controversia sobre qué tipo de paciente se beneficia más, manteniendo un coste-efectividad razonable. Varios estudio aleatorizados han demostrado un impacto positivo en la supervivencia y la calidad de vida de pacientes tratados con TAVIs a un coste aceptable ${ }^{30}$. Un estudio de coste-utilidad realizado en Reino Unido, expresado en QALYs (años de vida ajustados a calidad), demostró que a dos años, y teniendo en cuenta costes de ingreso, procedimiento, pruebas complementarias y seguimiento, la TAVI es una alternativa costeefectiva favorable en pacientes mayores operables y de alto riesgo ${ }^{39}$. Aunque hay publicados estudios en contra de la coste-efectividad de las TAVIs en el sistema de salud francés, estudios más recientes respaldan la coste-efectividad de este tipo de válvulas aludiendo una menor estancia hospitalaria tanto en UCl como en planta, debido a una movilización más preco ${ }^{40,41}$. Una conclusión recurrente en este tipo de estudios, es la importancia de una adecuada selección de pacientes, por lo que es obligatorio la conformación y discusión de cada caso por un HEART TEAM ${ }^{42-44}$.

\section{Conclusión}

Dados los múltiples avances en los cuidados médicos en las últimas décadas, la incidencia de la EA ha aumentado de forma significativa y paralelamente el envejecimiento poblacional, y es previsible que se mantenga esta tendencia. Por tanto, durante los próximos años, se tenderá a tratar a pacientes mayores y, por ello, con más comorbilidades asociadas. A la luz del estado actual y del desarrollo exponencial del tratamiento del paciente con EA severa, se debe individualizar la opción más apropiada para cada situación. En este sentido, cada paciente ha de ser discutido en una sesión clínica compuesta por un HEART TEAM, en la que clínicos, hemodinamistas y cirujanos tendrán en cuenta no sólo los factores de riesgo típicos de cada paciente, sino también factores tan diversos como su soporte social, capacidad funcional, esperanza de vida estimada, fragilidad, anatomía y otras comorbilidades, con intención de ajustar a cada individuo el mejor tratamiento posible en base a la evidencia científica disponible y a la experiencia de cada centro. Con la aparición de las TAVIs de segunda generación, las prótesis aórticas sin suturas y el desarrollo de procedimientos cada vez menos invasivos, la cirugía aortica convencional en pacientes mayores y con comorbilidades asociadas será cada 
vez menos frecuente en los próximos años. Sin embargo, faltan aún estudios que validen estas técnicas en pacientes de menos riesgo y con expectativa de vida larga, donde la cirugía convencional se ha mostrado como una técnica muy segura y con excelentes resultados a largo plazo.

\section{Bibliografía}

1. Osnabrugge RL, Mylotte D, Head SJ, Van Mieghem NM, Nkomo VT, LeReun CM, et al. Aortic stenosis in the elderly: disease prevalence and number of candidates for transcatheter aortic valve replacement: a meta-analysis and modeling study. J Am Coll Cardiol. 2013;62(11):1002-12.

2. Otto $\mathrm{CM}$, Prendergast $\mathrm{B}$. Aortic-valve stenosis--from patients at risk to severe valve obstruction. N Engl J Med. 2014;371(8):744-56.

3. Roberts WC, Ko JM. Frequency by decades of unicuspid, bicuspid, and tricuspid aortic valves in adults having isolated aortic valve replacement for aortic stenosis, with or without associated aortic regurgitation. Circulation. 2005;111(7):920-5.

4. Siu SC, Silversides CK. Bicuspid aortic valve disease. J Am Coll Cardiol. 2010;55(25):2789-800

5. Makkar RR, Fontana GP, Jilaihawi H, Kapadia S, Pichard AD, Douglas PS, et al. Transcatheter aortic-valve replacement for inoperable severe aortic stenosis. N Engl J Med. 2012;366(18):1696-704

6. Varadarajan P, Kapoor N, Bansal RC, Pai RG. Clinical profile and natural history of 453 nonsurgically managed patients with severe aortic stenosis. Ann Thorac Surg. 2006;82(6):2111-5

7. Varadarajan P, Kapoor N, Bansal RC, Pai RG. Survival in elderly patients with severe aortic stenosis is dramatically improved by aortic valve replacement: Results from a cohort of 277 patients aged $>$ or $=80$ years. Eur J Cardiothorac Surg. 2006;30(5):722-7.

8. Mathew V, Greason KL, Suri RM, Leon MB, Nkomo VT, Mack MJ, et al. Assessing the risk of aortic valve replacement for severe aortic stenosis in the transcatheter valve era. Mayo Clin Proc. 2014;89(10):1427-35.

9. lung B, Cachier A, Baron G, Messika-Zeitoun D, Delahaye F, Tornos P, et al. Decisionmaking in elderly patients with severe aortic stenosis: why are so many denied surgery? Eur Heart J. 2005;26(24):2714-20.

10. Bach DS, Cimino N, Deeb GM. Unoperated patients with severe aortic stenosis. J Am Coll Cardiol. 2007;50(20):2018-9

11. Vahanian A, Alfieri O, Andreotti F, Antunes MJ, Baron-Esquivias G, Baumgartner H, et al. Guidelines on the management of valvular heart disease (version 2012): the Joint Task Force on the Management of Valvular Heart Disease of the European Society of Cardiology (ESC) and the European Association for Cardio-Thoracic Surgery (EACTS). Eur J Cardiothorac Surg. 2012;42(4):S1-44

12. Nishimura RA, Otto CM, Bonow RO, Carabello BA, Erwin JP, Guyton RA, et al. 2014 AHA/ACC Guideline for the Management of Patients With Valvular Heart Disease: a report of the American College of Cardiology/American Heart Association Task Force on Practice Guidelines. Circulation. 2014;129(23):e521-643.

13. Brown ML, Schaff HV, Sarano ME, Li Z, Sundt TM, Dearani JA, et al. Is the European System for Cardiac Operative Risk Evaluation model valid for estimating the operative risk of patients considered for percutaneous aortic valve replacement? J Thorac Cardiovasc Surg. 2008;136(3):566-71.

14. Agarwal S, Tuzcu EM, Krishnaswamy A, Schoenhagen P, Stewart WJ, Svensson LG, et al. Transcatheter aortic valve replacement: current perspectives and future implications. Heart. 2014.

15. T C, F H. Cirugía cardiovascular en España en el año 2012. Registro de intervenciones de la Sociedad Española de Clrugía Torácica-Cardiovascular. Cirugia Cardiovascular. 2014;21:18-36

16. Bajona P, Suri RM, Greason KL, Schaff HV. Outcomes of surgical aortic valve replacement: the benchmark for percutaneous therapies. Prog Cardiovasc Dis. 2014;56(6):619-24

17. Sintek M, Zajarias A. Patient evaluation and selection for transcatheter aortic valve replacement: the heart team approach. Prog Cardiovasc Dis. 2014;56(6):572-82.

18. Rowe R, Iqbal J, Murali-Krishnan R, Sultan A, Orme R, Briffa N, et al. Role of frailty assessment in patients undergoing cardiac interventions. Open Heart. 2014;1(1):e000033.

19. Stortecky S, Schoenenberger AW, Moser A, Kalesan B, Jüni P, Carrel T, et al. Evaluation of multidimensional geriatric assessment as a predictor of mortality and cardiovascular events after transcatheter aortic valve implantation. JACC Cardiovasc Interv. 2012;5(5):489-96.

20. EIBardissi AW, Shekar P, Couper GS, Cohn LH. Minimally invasive aortic valve replacement in octogenarian, high-risk, transcatheter aortic valve implantation candidates. J Thorac Cardiovasc Surg. 2011;141(2):328-35.

21. Pope NH, Ailawadi G. Minimally Invasive Valve Surgery. J Cardiovasc Transl Res. 2014.

22. Khoshbin E, Prayaga S, Kinsella J, Sutherland FW. Mini-sternotomy for aortic valve replacement reduces the length of stay in the cardiac intensive care unit: metaanalysis of randomised controlled trials. BMJ Open. 2011;1(2):e000266.
23. Santarpino G, Pfeiffer S, Jessl J, Dell'Aquila AM, Pollari F, Pauschinger M, et al. Sutureless replacement versus transcatheter valve implantation in aortic valve stenosis: a propensity-matched analysis of 2 strategies in high-risk patients. J Thorac Cardiovasc Surg. 2014;147(2):561-7.

24. D'Onofrio A, Messina A, Lorusso R, Alfieri OR, Fusari M, Rubino P, et al. Sutureless aortic valve replacement as an alternative treatment for patients belonging to the "gray zone" between transcatheter aortic valve implantation and conventional surgery: a propensity-matched, multicenter analysis. J Thorac Cardiovasc Surg. 2012;144(5):1010-6.

25. Kocher AA, Laufer G, Haverich A, Shrestha M, Walther T, Misfeld M, et al. One-year outcomes of the Surgical Treatment of Aortic Stenosis With a Next Generation Surgical Aortic Valve (TRITON) trial: a prospective multicenter study of rapid-deployment aortic valve replacement with the EDWARDS INTUITY Valve System. J Thorac Cardiovasc Surg. 2013;145(1):110-5; discussion 5-6

26. Santarpino G, Pfeiffer S, Schmidt J, Concistre G, Fischlein T. Sutureless aortic valve replacement: first-year single-center experience. Ann Thorac Surg. 2012;94(2):5048; discussion 8-9.

27. Sepehripour AH, Harling L, Athanasiou T. What are the current results of sutureless valves in high-risk aortic valve disease patients? Interact Cardiovasc Thorac Surg. 2012;14(5):615-21.

28. Forcillo J, Pellerin M, Perrault LP, Cartier R, Bouchard D, Demers P, et al. CarpentierEdwards pericardial valve in the aortic position: 25-years experience. Ann Thorac Surg. 2013;96(2):486-93.

29. Cribier A, Eltchaninoff H, Bash A, Borenstein N, Tron C, Bauer F, et al. Percutaneous transcatheter implantation of an aortic valve prosthesis for calcific aortic stenosis: first human case description. Circulation. 2002;106(24):3006-8.

30. Leon MB, Smith CR, Mack M, Miller DC, Moses JW, Svensson LG, et al. Transcatheter aortic-valve implantation for aortic stenosis in patients who cannot undergo surgery. N Engl J Med. 2010;363(17):1597-607.

31. Eggebrecht H, Schmermund A, Voigtlander T, Kahlert P, Erbel R, Mehta RH. Risk of stroke after transcatheter aortic valve implantation (TAVI): a meta-analysis of 10,037 published patients. Eurolntervention. 2012;8(1):129-38.

32. Neragi-Miandoab S, Michler RE. A review of most relevant complications of transcatheter aortic valve implantation. ISRN Cardiol. 2013;2013:956252.

33. Thomas M, Schymik G, Walther T, Himbert D, Lefèvre T, Treede H, et al. Thirty-day results of the SAPIEN aortic Bioprosthesis European Outcome (SOURCE) Registry: A European registry of transcatheter aortic valve implantation using the Edwards SAPIEN valve. Circulation. 2010;122(1):62-9.

34. Erkapic D, De Rosa S, Kelava A, Lehmann R, Fichtlscherer S, Hohnloser SH. Risk for permanent pacemaker after transcatheter aortic valve implantation: a comprehensive analysis of the literature. J Cardiovasc Electrophysiol. 2012;23(4):391-7.

35. Stortecky S, Wenaweser P, Diehm N, Pilgrim T, Huber C, Rosskopf AB, et al. Percutaneous management of vascular complications in patients undergoing transcatheter aortic valve implantation. JACC Cardiovasc Interv. 2012;5(5):515-24.

36. Chiam PT, Ewe SH. An update on complications associated with transcatheter aortic valve implantation: stroke, paravalvular leak, atrioventricular block and perforation. Future Cardiol. 2013;9(5):733-47

37. Finn M, Green P. Transcatheter aortic valve implantation in the elderly: who to refer? Prog Cardiovasc Dis. 2014;57(2):215-25.

38. Davies WR, Thomas MR. European experience and perspectives on transcatheter aortic valve replacement. Prog Cardiovasc Dis. 2014;56(6):625-34.

39. Fairbairn TA, Meads DM, Hulme C, Mather AN, Plein S, Blackman DJ, et al. The costeffectiveness of transcatheter aortic valve implantation versus surgical aortic valve replacement in patients with severe aortic stenosis at high operative risk. Heart. 2013:99(13):914-20

40. Chevreul K, Brunn M, Cadier B, Haour G, Eltchaninoff H, Prat A, et al. Cost of transcatheter aortic valve implantation and factors associated with higher hospital stay cost in patients of the FRANCE (FRench Aortic National CoreValve and Edwards) registry. Arch Cardiovasc Dis. 2013;106(4):209-19.

41. Prendergast BD, Naber CK, Popma JJ. Transatlantic perspectives on TAVI: from essential infrastructure and integration to expansion, research and development. Heart. 2012;98 Suppl 4:iv37-43.

42. Puñal Riobóo J, De la Fuente Cid R. Eficacia y seguridad del implante valvular percutáneo/transapical en el tratamiento de la estenosis aórtica grave. Red Española de Agencias de Evaluación de Tecnologías y Prestaciones del SNS Axencia de Avaliación de Tecnoloxías Sanitarias de Galicia Informes de evaluación de tecnologías sanitarias. 2013.

43. Puñal Riobóo J, De la Fuente Cid R, Queiro Verdes T, Varela Lerna L, López-García M. Desarrollo de indicadores de calidad para el implante transcatéter de prótesis valvular aórtica en el tratamiento de la estenosis aórtica grave. Consenso de expertos. Red Española de Agencias de Evaluación de Tecnologías y Prestaciones del SNS Axencia de Avaliación de Tecnoloxías Sanitarias de Galicia Informes de tecnologías sanitarias. 2013

44. Queiro Verdes T, López-García M, Puñal Riobóo J, De la Fuente Cid R, Varela-Lema L, Cequier Fillat Á. Desarrollo de indicaciones de uso apropiado del implante transcatéter de prótesis valvular aórtica (TAVI) en el tratamiento de la estenosis aórtica grave sintomática. Red Española de Agencias de Evaluación de Tecnologías y Prestaciones del SNS Axencia de Avaliación de Tecnoloxías Sanitarias de Galicia Informes de tecnologías sanitarias. 2014. 